\title{
Le dibi-madibi : un mode de faire-valoir original dans le sud-ouest du Togo
}

The dibi-madibi: an original mode of tenure in Southwestern Togo

\section{Padabô Kadouza}

\section{(2) OpenEdition}

\section{Édition électronique}

URL : http://journals.openedition.org/belgeo/6510

DOI : $10.4000 /$ belgeo.6510

ISSN : 2294-9135

Éditeur :

National Committee of Geography of Belgium, Société Royale Belge de Géographie

\section{Édition imprimée}

Date de publication : 1 octobre 2010

Pagination : 331-341

ISSN : 1377-2368

\section{Référence électronique}

Padabô Kadouza, "Le dibi-madibi : un mode de faire-valoir original dans le sud-ouest du Togo »,

Belgeo [En ligne], 3 | 2010, mis en ligne le 15 décembre 2012, consulté le 01 mai 2019. URL : http:// journals.openedition.org/belgeo/6510; DOI : 10.4000/belgeo.6510

Ce document a été généré automatiquement le 1 mai 2019.

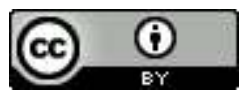

Belgeo est mis à disposition selon les termes de la licence Creative Commons Attribution 4.0 International. 


\title{
Le dibi-madibi : un mode de faire-valoir original dans le sud-ouest du Togo
}

The dibi-madibi: an original mode of tenure in Southwestern Togo

\author{
Padabô Kadouza
}

\section{Introduction}

1 Le sud-ouest du Togo (figure 1) est une région qui bénéficie de conditions naturelles propices aux cultures de plantation. Au début de sa mise en valeur, le "dibi-madibi" qui signifie à la lettre en Twi (une langue du Ghana) "tu manges un peu, je mange un peu" a permis de résoudre le problème de main-d'oeuvre et d'assurer une rapide conquête de l'espace. Le dibi-madibi est un mode de faire valoir par lequel un propriétaire d'un terrain en friche fait mettre en valeur par un tiers ledit terrain dans le but de lui céder la moitié avec un droit de propriété. Le principe de base est que le terrain complanté n'appartient ni au propriétaire tout seul, ni à l'exploitant tout seul. Il appartient aux deux qui le partagent équitablement. Le problème que soulève cette pratique et de savoir quelle est sa nature juridique et comment peut-on la qualifier en géographie ? Cette étude vise deux objectifs :

- déterminer les natures juridique et géographique du dibi-madibi ;

- dégager les problèmes qu'il entraîne entre propriétaires terriens et exploitants.

Pour atteindre ces objectifs, nous avons d'abord, fait une recherche documentaire dans laquelle une importance a été accordée aux ouvrages de droit civil, de droit rural et foncier. Ensuite, des interviews qui se sont basées sur les aires culturelles de la zone d'étude ont été réalisées. Au total, 70 paysans (25 en pays Akposso, 20 en zone Akébou et 35 dans l'aire culturelle Ewé) ont été interviewés. Le choix de ces paysans a été fait de façon aléatoire en respectant la parité entre les propriétaires terriens ou leurs descendants et les exploitants. 
Figure 1. Localisation du sud-ouest du Togo.

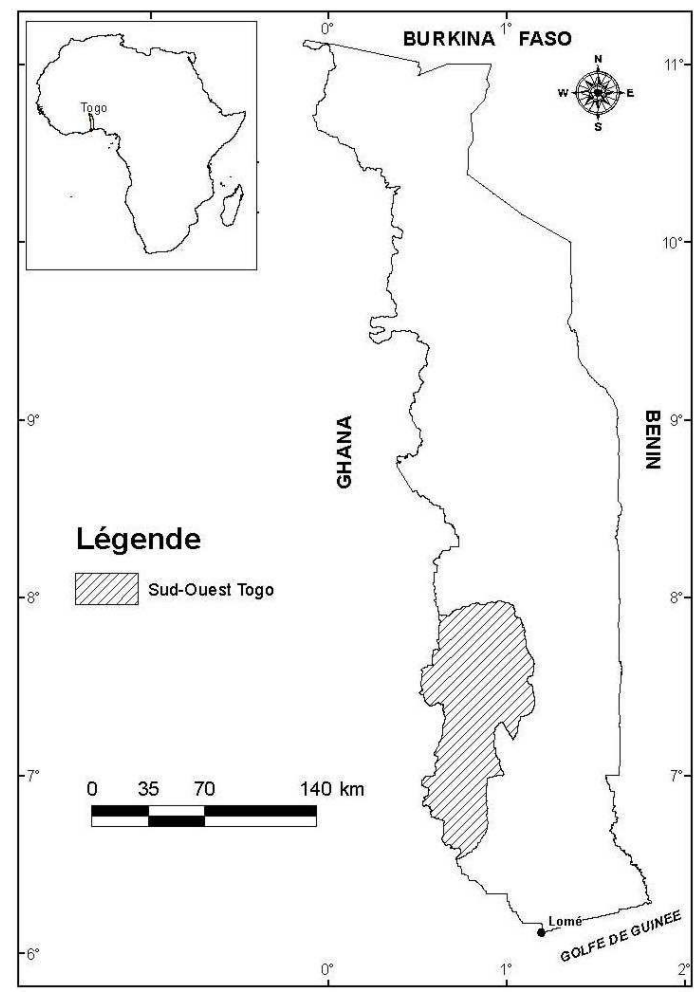

Source : D’après Atlas Jeune Afrique - Togo, 1981

\section{Le Sud-Ouest : une région favorable aux cultures de plantation}

La région qui fait l'objet de cette étude représente la partie de la chaîne des monts du Togo située au sud du parallèle $8^{\circ} \mathrm{N}$ où les altitudes les plus élevées atteignent en moyenne $800 \mathrm{~m}$, avec pour point culminant le mont Dzogadzeto $(972 \mathrm{~m})$. Cette partie se trouve dans le prolongement méridional de la chaîne de l'Atacora qui prend en écharpe le pays. Elle s'étend sur près de $7500 \mathrm{~km} 2$ soit $13 \%$ du territoire et regroupe environ $15 \%$ de la population du pays. Sur le plan administratif, elle couvre sept préfectures (Amou, Wawa, Kloto, Agou, Kpélé, Danyi, Akébou). Les groupes ethniques dominants dans ces préfectures sont les Ewé, les Akposso et les Akébou, installés dans la région depuis le XVIIe (Gu-Konu, 1978). Mais, le fait humain le plus caractéristique du milieu est la forte attraction qu'il a exercée depuis le début de l'économie de plantation, sur les populations des autres régions, surtout celles densément peuplées du nord du pays au climat impropre à la culture du caféier et du cacaoyer. Depuis le début du processus colonial, le fait dominant de l'économie régionale est l'activité de plantation, axée sur la culture du caféier et du cacaoyer (la région fournit $90 \%$ du café et presque la totalité du cacao produit par le Togo). "Mais, le secteur vivrier subsiste et intéresse sous deux angles: primo, il produit de plus en plus pour le marché et diversifie par ce fait, les sources de revenu monétaire du paysan; secundo, il représente sans cesse le domaine d'une série d'expériences en vue de la modernisation de l'agriculture. Ces deux faits sont étroitement liés par une série de relations dont le noeud se trouve dans l'introduction et l'extension 
de l'économie de marché dans la région par le moyen des plantations paysannes" (GuKonu, 1978).

La nature et la diversité du milieu écologique sont les facteurs déterminants de cette activité de plantation. Sur le plan physique, la région est un plateau schisto-quartzitique disséqué par un réseau hydrographique dense. Le résultat en est un modelé de collines et de lambeaux de surfaces tabulaires séparés par des vallées profondes. Cette évolution morphologique a créé des conditions édaphiques propices à la culture du caféier et du cacaoyer. La région bénéficie d'un climat subéquatorial de montagne avec des pluies abondantes qui atteignent $1700 \mathrm{~mm}$ par an. La saison sèche se caractérise par un à deux mois écologiquement secs avec des précipitations inférieures à $20 \mathrm{~mm}$ au mois de janvier (figure 2). Cette saison sèche est également marquée par un faible déficit de saturation et par l'abondance des brouillards matinaux qui ne traduisent que des précipitations occultes (Gnongbo, 2003). La température moyenne mensuelle oscille autour de $25^{\circ} \mathrm{C}$, avec des maxima qui atteignent $32^{\circ} \mathrm{C}$ et des minima qui tombent jusqu'à $15^{\circ} \mathrm{C}$. La faiblesse de l'évapotranspiration contribue au maintien de l'humidité de l'air, ce qui se traduit par une hygrométrie permanente de l'ordre de 70 à $90 \%$. Ces caractéristiques sont des facteurs ayant contribué au maintien d'une forêt dense humide qui est un héritage de la grande forêt intertropicale africaine bien entretenue par l'isopluvial du maximum nouakchottien (6000 à 5500 ans B P) à un moment où la pluviométrie était mieux répartie (Akpagana, 1989 ; Gnongbo, 1996). La combinaison de l'abondance pluviométrique et de la forte capacité de rétention en eau, ainsi que les produits de décomposition des schistes et des quartzites offrent au caféier et au cacaoyer les conditions d'humidité satisfaisantes pour leur développement.

5 En définitive, le milieu naturel du sud-ouest du Togo, par ses traits morphologiques et par ses caractères bioclimatiques offre un cadre favorable pour le développement de la culture du caféier et du cacaoyer. 
Figure 2. Diagramme ombrothermique de Kouma-Konda (1961-2009).

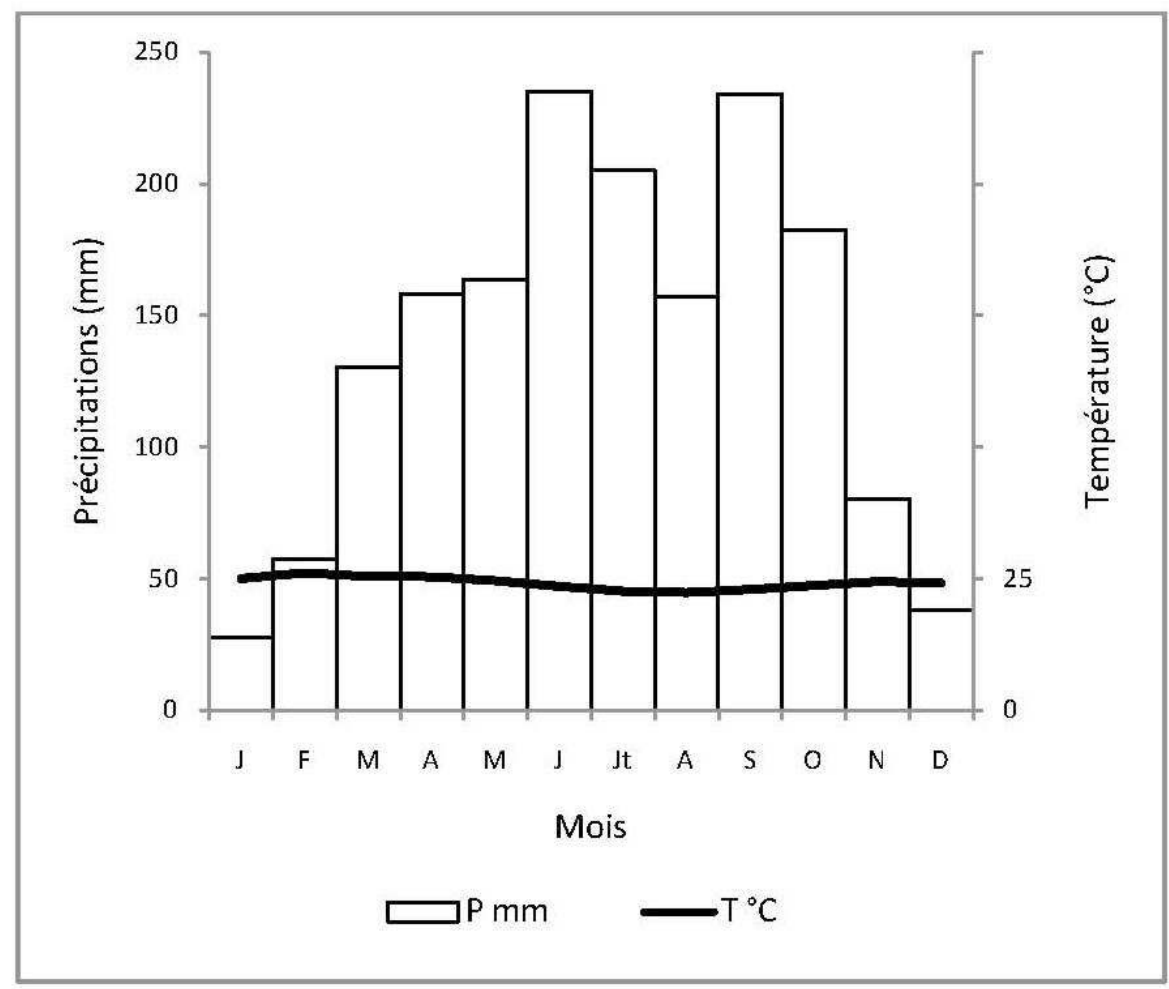

Source : D’après les données de la Direction de la Météorologie nationale

\section{Le dibi-madibi : une stratégie d'appropriation du sol et de l'exploitation}

Avant l'apparition de l'économie de plantation dans le sud-ouest togolais, l'activité agricole était axée sur la production de céréales (maïs, riz, fonio) et de tubercules (manioc, igname, taro) qui étaient les produits de base du régime alimentaire. Cette agriculture de subsistance se faisait dans le cadre d'un mode de production traditionnel avec des moyens techniques limités pour l'essentiel à la houe, au coupe-coupe et au feu (culture itinérante sur brûlis). "Cette faiblesse du capital technique a conféré à la solidarité du groupe social une place fondamentale dans la logique de l'organisation paysanne; les pratiques communautaires découlant de cette solidarité, bien qu'informelles, étaient devenues pour l'individu, le seul moyen efficace de surmonter, au cours du procès de production, les difficultés liées à la faiblesse du niveau technique" (GuKonu, 1978). Ces moyens techniques à faible productivité n'ont pu permettre que des exploitations de petite taille. En effet, les exploitations de moins d'un hectare représentaient $33 \%$ du nombre total des exploitations dans la circonscription administrative de Kloto, et si l'on considère les exploitations de moins de deux hectares, la proportion monte alors à $66,5 \%$ (Gu-Konu, 1983).

7 L'appropriation de la terre en tant que condition de l'activité agricole se faisait dans le cadre du lignage ou de la famille. C'est dans ce cadre socio-économique qu'apparut dans la zone d'étude au début du xxe siècle, l'économie de plantation fondée sur la culture du caféier et du cacaoyer. Introduite par les missionnaires allemands venus de Brême, ces 
cultures firent très vite l'objet d'un programme systématique de diffusion par l'administration allemande entre 1890 et 1900. Elles furent pratiquement imposées à la paysannerie locale dans l'entre-deux-guerres par l'administration mandataire ${ }^{1}$ française. C'est toutefois après la seconde guerre mondiale que les plantations paysannes vont prendre un véritable essor, sous l'incitation d'une politique généralisée de hauts prix d'achat.

\section{La pratique du dibi- madibi}

Le dibi-madibi est un contrat très formaliste, bien que souvent non écrit. Il n'est valable que pour les terrains destinés aux cultures pérennes (café et cacao notamment).

9 Avant la conclusion du contrat, les parties accompagnées de quelques témoins se rendent sur le terrain à mettre en valeur. Le propriétaire foncier indique au futur exploitant ses limites. Après cette visite des lieux, on fait des sacrifices aux mânes des ancêtres et le contrat est conclu. Ces sacrifices traduisent si bien ce que dit un proverbe Agni de Côted'Ivoire : "Ce n'est pas l'homme qui possède la terre, c'est la terre qui possède l'homme" (Verdier, 1986). En effet, les peuples de la zone d'étude (Ewé, Akposso et Akébou), à l'instar des autres Africains au sud du Sahara, considèrent qu'avant d'être un espace que l'homme s'approprie, la terre est d'abord une entité spirituelle à laquelle il appartient. Aussi, la prise de possession d'une terre inoccupée s'accompagne-t-elle de libations non seulement à elle, mais aussi aux ancêtres qui en sont les vrais propriétaires.

Après la conclusion du contrat, le futur exploitant, fournit des boissons ${ }^{2}$ et parfois des mets qui sont consommés par toute l'assistance. Une fois ces formalités remplies, on estime le contrat définitivement conclu entre le propriétaire terrien (Agbleto) et l'exploitant (Apavi). A l'origine, il s'agissait d'un contrat oral, mais depuis le début des années 1970, on a pris l'habitude de le rédiger en y mentionnant la situation géographique du terrain, ses limites et si possible ses dimensions.

11 La durée de la période intermédiaire (période qui sépare la conclusion du contrat du partage du terrain) est globalement d'une dizaine d'années. En effet, le principe est que le partage du terrain est réalisé après que la plantation commence à produire. Or, la pratique veut que le partage ne soit fait que trois ans après la première récolte, laquelle n'intervient que six ou sept ans après la complantation du terrain. La conséquence est que la période intermédiaire dure neuf ou dix ans. Une plantation créée en 2010 suivant le contrat du dibi-madibi ne sera partagée entre le propriétaire foncier et l'exploitant qu'en 2019 ou 2020. Mais cette durée peut varier. Les éléments dont on se sert dans la détermination de ce délai sont non seulement la date de création de la plantation, mais aussi et surtout le moment de production du verger. Il en résulte que cette durée peut être écourtée si le verger produit plus rapidement que d'habitude ou être rallongée si compte tenu des conditions écologiques, il produit plus tardivement.

12 Les droits et obligations pendant cette période sont de deux types:

- les droits et obligations du propriétaire terrien;

- ceux de l'exploitant.

13 Concernant les droits et les obligations du propriétaire terrien, il ne doit rien faire qui puisse gêner l'exploitant dans l'exécution du contrat. Il doit le protéger contre les propriétaires voisins (que l'on désigne dans la région sous le vocable de "limitrophes"). Il doit mettre à sa disposition un autre terrain sur lequel il pratiquera durant la période 
intermédiaire les cultures vivrières. Les exploitants étant presque tous des immigrants (Kabyè, Nawdéba, Lamba etc.), ceux-ci ont besoin de moyens de subsistance pour accomplir le contrat dans de bonnes conditions. Pour Gu-Konu (1983), cette clause répond à la nécessité de la reproduction de la force de travail du travailleur qui se confond avec le traditionnel droit de tout individu, qu'il soit étranger au village ou non, de disposer d'une parcelle pour sa survie, conformément au principe que la terre est avant tout un moyen pour vivre et non un moyen pour accumuler les richesses.

Concernant l'exploitant, sa principale obligation est de créer la plantation et de bien l'entretenir jusqu'à ce qu'elle commence à produire.

Pendant les premières années de la plantation, il peut associer la culture du café ou du cacao à des cultures vivrières (manioc, taro, bananes, maïs) sans que ces dernières fassent l'objet de partage entre lui et le propriétaire foncier. Enfin, il a le droit de jouir des produits de la plantation pendant trois ans à partir de la première récolte, avant le partage du terrain.

A la fin de la période intermédiaire, intervient le partage du terrain. L'initiative en revient à l'exploitant qui déclenche les formalités en apportant au propriétaire terrien certains bien dits de "remerciement". Ces biens comprennent essentiellement une partie de la dernière récolte faite par l'exploitant et quelques bouteilles de sodabi et de boissons fortes importées. Après la remise de ces biens, on procède au partage de la parcelle en deux parties égales. Le partage est fait par les témoins du propriétaire foncier et ceux de l'exploitant après avoir mesuré les dimensions de la parcelle. Une fois le partage réalisé, c'est le propriétaire terrien qui choisit d'abord la partie qu'il veut. D’une façon générale, l'exploitant prend la partie où se trouve son habitation. Il acquiert en pleine propriété la moitié du terrain qu'il a mis en valeur. De simple exploitant qu'il était, il devient propriétaire foncier. En plus de cette partie qui lui revient, il arrive souvent qu'il prenne en charge la moitié restante de la parcelle suivant la formule du partage de la récolte annuelle.

\section{Les natures juridique et géographique du contrat du dibi-madibi}

\section{La nature juridique du contrat}

17 La détermination de la nature juridique du dibi-madibi soulève une question importante. S'agit-il d'un contrat d'exploitation ou d'un contrat d'appropriation ?

Au Togo, il existe des contrats d'exploitation par lesquels le titulaire du droit de culture, au lieu d'exploiter lui-même sa terre, peut confier l'exploitation à un tiers. On en distingue quatre. Il ya d'abord le prêt et le gage. Le prêt est un contrat essentiellement gratuit et temporaire qui est en principe uniquement utilisé pour les cultures vivrières. Par le prêt, le "prêteur" ne confère à l'emprunteur que le droit d'usage d'un terrain. Il en est de même pour le gage par lequel l'exploitant obtient uniquement l'usage du bien mis en gage. Le dibi-madibi opérant le transfert de la propriété de la moitié du terrain complanté, il ne peut être comparé à ces deux contrats. Il y a ensuite le contrat de louage qui est selon l'article 1709 du code civil "un contrat par lequel l'une des parties s'oblige à faire jouir l'autre d'une chose pendant un certain temps, et moyennant un certain prix que celle-ci s'oblige de lui payer". Ceci étant, le contrat du dibi-madibi dont la finalité est 
d'aboutir à la cession de la moitié du terrain à l'exploitant ne peut être assimilé à celui du louage dans la mesure où il n'y a pas de versement de redevance soit en nature soit en espèces.

19 Concernant le contrat d'appropriation, les coutumes foncières togolaises reconnaissent également des contrats qui transfèrent le droit de propriété. Parmi ces contrats, nous pouvons citer la donation, la vente et l'échange. Le dibi-madibi ne peut pas être assimilé à la donation, puisque ce contrat est essentiellement gratuit. Il ne peut être considéré comme la vente, car la vente est la convention par laquelle une des parties s'oblige à livrer une chose et l'autre à payer le prix. S'agit-il d'un échange? Selon l'article $1702 \mathrm{du}$ code civil, "l'échange est un contrat par lequel, les parties se donnent respectivement une chose pour une autre". Le dibi-madibi étant un contrat qui aboutit à la cession en pleine propriété de la moitié du terrain, objet du contrat, l'exploitant devra mettre en valeur ledit terrain. Il doit le complanter soit de cacaoyers soit de caféiers. Il doit entretenir la plantation jusqu'à ce que cette dernière commence à produire. L'exploitant remet à la fin d'une période donnée une exploitation au propriétaire foncier. De son côté, le propriétaire fournit à l'exploitant une partie du terrain. Dans ces conditions on peut admettre qu'il s'agit d'un échange. Ainsi analysé, le contrat du dibi-madibi répond à la définition de l'échange. Mais, si on considère que le contrat $a b$ initio porte sur tout le terrain, cette conclusion doit être nuancée en raison de l'assiette du contrat. Il paraitt donc difficile d'analyser ce contrat en termes d'échange dans sa phase initiale. Si on peut parler d'échange, ce ne peut être que dans la phase finale du contrat. Il s'agit donc d'un contrat spécial qui ne trouve son pareil dans les contrats traditionnels togolais. Le dibimadibi serait un contrat sui generis.

\section{Comment qualifier le contrat du dibi-madibi en géographie ?}

Le dibi-madibi est en géographie un mode de faire-valoir indirect, puisque le propriétaire ne met pas lui-même la terre en valeur. Mais, le problème qui se pose est de savoir si ce contrat est le fermage ou le métayage. En effet, le fermage est un mode de faire-valoir où le propriétaire foncier loue son bien à un fermier qui acquiert le droit d'exploiter pendant la durée d'un bail contre une somme d'argent. Ceci étant, le dibi-madibi n'est pas le fermage. Qu'en est-il du métayage ? Le métayage est un type de bail rural dans lequel, un propriétaire (le bailleur) confie à un métayer le soin de cultiver une terre en échange d'une partie de la récolte. George et Verger (1996) en donnent une définition plus précise: "le métayage est un mode de faire-valoir où le propriétaire et l'exploitant (métayer) se partagent les produits, en principe par moitié".

Ces deux définitions rapprochent le contrat du dibi-madibi du métayage. Mais, si on considère le contrat dans sa phase finale, il ne peut être assimilé au métayage dans le sens occidental du terme, puisque le résultat final est le partage du terrain et non les produits. C'est pourquoi, Gu-Konu (1983) dit que le dibi-madibi est sommairement identifié au métayage. Nous pouvons donc dire que le dibi-madibi est un mode de faire-valoir indirect original. Ce type de contrat se pratique également dans les zones de plantations ghanéennes (partie orientale du Ghana) dont il est d'ailleurs originaire et dans la région du Bas-Sassandra en Côte d'Ivoire. Les informations recueillies font état d'une similitude entre le dibi-madibi pratiqué au sud-ouest togolais et celui de l'est ghanéen. Quant au dibi-madibi de Côte-d'Ivoire, apparu au milieu des années 1980 avec la disparition des disponibilités forestières d'une part et l'affaiblissement de l'Etat d'autre part, on note une 
certaine différence qui réside dans le fait qu'il correspond à la construction de rente foncière. En effet, l'étude réalisée par Léonard et Vimard (2005), a révélé que "si l'on considère les arrangements en dibi-madibi, les charges de mise en place d'un verger transférées au demandeur de terre, représentaient en 1995, un coût de 150 à 175000 FCFA, soit un niveau très supérieur au prix d'hectare de forêt cédé en contrepartie"3. Le contrat du dibi-madibi dans le sud-ouest togolais soulève depuis un certain temps, des problèmes entre propriétaires terriens et exploitants. L'ampleur de ces problèmes mérite qu'on s'attarde un peu sur eux.

\section{Les problèmes posés par le dibi-madibi}

Le contrat du dibi-madibi entraîne dans certaines localités des litiges qui naissent le plus souvent des contestations des descendants d'anciens propriétaires terriens. Ceux-ci acceptent mal l'acquisition en pleine propriété de la moitié de la parcelle mise en valeur par les exploitants. Ils auraient préféré que ce soit la plantation qui soit partagée. Ces litiges qui remontent aux années 1970 selon les résultats de nos interviews ont été exacerbés par les troubles socio-politiques ${ }^{4}$ qu'a connus le Togo au début des années 1990. Beaucoup d'anciens propriétaires fonciers ou leurs descendants profitèrent de cette situation d'instabilité pour remettre en cause le contrat du dibi-madibi.

En effet, ils réclamèrent les parcelles qui revenaient aux exploitants après le partage sous prétexte que le contrat ne concerne que les arbres plantés et non la terre.

C'est ainsi que dans certains villages comme Klabè-Efoukpa, Djon- Kotora, Sérégbénè, des exploitants furent menacés et leurs parcelles retirées. Ces derniers regagnèrent pour l'essentiel les Régions Centrale et de la Kara où ils s'adonnent aujourd'hui aux cultures vivrières. Ils sont désignés sous le nom de "Poronamba" (ce qui signifie les chassés).

D'autres, sans informer les exploitants, allèrent couper sur leurs parcelles, les arbres utiles (Milicia excelsa, Antiaris africana, Khaya senegalensis, Cola gigantea) très utilisés dans la région pour la fabrication des planches qui servent à faire des meubles. Dans des localités comme Klabè-Soto, Dzogbégan, Otandjobo, certains descendants des propriétaires terriens ont investi les champs des exploitants soit pour récolter les noix de palme soit pour abattre les palmiers dans le but de fabriquer le vin de palme. A travers ces actions, ils voulurent "réaffirmer" leurs droits sur ces parcelles. A qui veut savoir le pourquoi, ils lui répondent que les exploitants n'ont pas apporté la terre de chez eux dans le sud-ouest. D'autres formulèrent de nouvelles exigences inattendues envers leurs exploitants dans le but de les obliger à renégocier le contrat en apportant encore des biens (boissons et même animaux pour sacrifice aux ancêtres). Les interviews que nous avons faites nous ont permis de connaître les motivations de ces propriétaires ou de leurs descendants. Ces motivations peuvent être regroupées en trois principales idées.

D'abord, ils pensent que la terre du lignage ou de la famille est destinée à la reproduction du groupe. Elle doit subvenir aux besoins de ses membres présents et futurs. Elle ne doit pas sortir du groupe et faire l'objet d'appropriations privatives qui permettraient à quelques individus de l'accaparer. Ensuite, ils pensent également qu'en cédant la moitié de la parcelle à l'exploitant selon le contrat de dibi- madibi, il s'agit d'un transfert précaire qu'on peut annuler à tout moment. Enfin, ils ont le sentiment que le caractère imprescriptible et l'inaliénabilité de la terre en pays Ewé, Akposso et Akébou ne peuvent pas permettre de la céder aux étrangers de façon définitive. Cette manière de penser de 
ces propriétaires terriens du sud-ouest du Togo rejoint celle des Ibo du Nigéria et des Dogon du Mali chez qui même la vente de la terre (avec la monétarisation de l'économie) n'est jamais définitive et qu'on peut la résilier en restituant le prix (Verdier, 1986).

Les litiges de plus en plus fréquents et l'épuisement des surfaces ${ }^{5}$ plantables ont progressivement fait passer ce régime de partage de la parcelle à celui du produit (Abusa), le rôle des nouveaux "métayers" n'étant plus de créer la plantation, mais d'assurer l'entretien et la récolte d'un verger déjà existant, avec pour rétribution une part de la production. Cette part peut varier dans l'espace en fonction de l'âge du verger et des rapports de force entre le "métayer" et le propriétaire terrien. Dans le Litimé, dominé par la culture du cacao, les personnes interviewées avancent une part de $1 / 4$ ou $1 / 5$, alors que sur le plateau Akposso, zone de prédilection du café, il est de $1 / 3$. D'autres contrats occultés pendant longtemps par le dibi-madibi connaissent de plus en plus un regain d'intérêt. Il s'agit notamment du kotokouanou et de awoba. S'agissant du kotokouanou, le migrant entretient un verger et en assure la récolte. Sa rémunération est fonction du nombre de sacs récoltés. Il reçoit en prêt une habitation et des parcelles pour la culture des vivriers et dispose de temps libre pour s'adonner à d'autres activités. Il n'est pas rare qu'un allochtone ayant bénéficié d'un contrat de kotokouanou travaille chez plusieurs planteurs, multipliant ainsi revenus et parcelles de vivriers. Pour ce qui concerne awoba, le propriétaire met son verger en gage contre une somme fixe pendant un certain nombre d'années (généralement pas plus de cinq ans). On n'enregistre pratiquement plus dans la zone d'étude l'ouverture de nouvelles plantations. Le "vieux" contrat du dibi-madibi ayant permis la dynamique pionnière du sud-ouest du Togo et qui a connu de beaux jours connaît aujourd'hui des mutations remarquables.

Les problèmes que pose le dibi-madibi conjugués avec la crise que connaît la filière cafécacao aujourd'hui ont émoussé l'ardeur au travail de beaucoup d'immigrants Kabyè, Nawdéba et Lamba, "vrais créateurs de plantations", qui se tournent de plus en plus vers les cultures vivrières.

\section{Conclusion}

Le dibi-madibi, pratique agraire originale, a facilité la mise en valeur coloniale du sudouest du Togo, région où l'environnement bioclimatique est propice aux cultures de plantation. Il a permis de pallier le problème de main-d'oeuvre agricole avec l'évolution des structures sociales. Cette évolution a eu des conséquences importantes sur les modes d'exploitation communautaires $\mathrm{du}$ sol qui firent progressivement place à l'individualisation du travail. L'analyse de la nature du dibi-madibi a permis de savoir qu'il ne peut être comparé à aucun autre contrat traditionnel connu au Togo sur le plan juridique. Au plan géographique, il ne se confond ni au fermage ni au métayage classiques où on note des rapports de soumission. Bien que le dibi-madibi soit un mode de faire valoir qui ait permis aux immigrants d'avoir des droits de propriété sur la moitié des parcelles exploitées, il crée aujourd'hui des problèmes entre anciens propriétaires et exploitants. Pour éviter que ces problèmes n'entravent le développement du sud-ouest, déjà mis en mal par la crise caféière et cacaoyère, il s'avère nécessaire que les autorités locales (préfets, chefs traditionnels etc.) et les responsables régionaux du Ministère de l'Agriculture, de l'Elevage et de la Pêche fassent une sensibilisation à l'endroit des descendants d'anciens propriétaires terriens sur la nécessité de respecter les clauses du contrat, afin de garantir de bonnes relations avec les immigrés. 


\section{BIBLIOGRAPHIE}

ADJOSSOU K. (2009), Caractérisation de la biodiversité dans les forêts tropicales humides du Togo, Thèse unique de doctorat, spécialité Botanique et Ecologie végétale, Faculté des Sciences, Université de Lomé, 210 p. + annexes.

AFFOU-YAPI S. (1979), Le grand planteur villageois dans le procès de valorisation du capital social. Une introduction à l'organisation socio-économique akyé, ORSTOM, Abidjan, 360 p.

AKPAGANA K. (1989), Recherches sur les forêts denses humides du Togo, Université de Bordeaux III, UFR Aménagement et Ressources Naturelles, Thèse de doctorat Nouveau Régime, 181 p.

BACHELET M. (1968), Système foncier et réformes agraires en Afrique Noire, Paris, Librairie Générale de droit et de jurisprudence, $677 \mathrm{p}$.

BASSA K. O. (1996), Contribution à l'histoire des Akposso : “les Logbo”, Mémoire de Maîtrise d'Histoire, Département d'Histoire, Faculté des Lettres et Sciences Humaines, Université de Lomé, $101 \mathrm{p}$.

CORNEVIN R. (1962), Histoire du Togo, Paris, Edition Berger-Levrault, 2e édition, Collection d'Outre-Mer, $427 \mathrm{p}$.

FOLI M. L. (1970), Le régime juridique de la terre au Togo, Thèse de doctorat en droit, Université de Paris I-Sorbonne, $423 \mathrm{p}$.

GEORGE P. et VERGER F. (1996), Dictionnaire de la Géographie, Paris, PUF, 500 p.

GNONGBO T. Y. (1996), Le Togo méridional :étude de géographie physique, Thèse de doctorat de Géographie physique tropicale, Université de Bordeaux III, 306 p.

GNONGBO T. Y. (2003),"Mise en valeur agricole et évolution du milieu naturel dans la zone forestière du Litimé (Togo)”, Les Cahiers d'Outre-Mer, 224, octobre- décembre. Mis en ligne le 13 février 2008.

GU-KONU E. Y. (1978), “Plantations paysannes et stratégies de l'espace dans le sud-ouest du Togo", in Maîtrise de l'espace agraire et développement en Afrique. Logique paysanne et rationalité technique, Actes du colloque de Ouagadougou du 4 au 8 décembre, pp. 93-103.

GU-KONU E. Y. (1983), Tradition et modernité : la modernisation agricole face à la mutation rurale en Afrique Noire : l'exemple du Togo : la mutation rurale et la logique de la société de transition, 3 vol. , Thèse de doctorat d'Etat de Géographie, Paris I- Sorbonne, 1037 p.

GUILLIEN R. et VINCENT J. (2003) (dir.), Lexique des termes juridiques, Paris, Dalloz, 14e édition, $619 \mathrm{p}$.

HENRY X. et al., (2005), Code civil, Paris, Dalloz, 104e édition, 2269 p.

KOUASSIGAN G. A. (1966), L'homme et la terre: droits fonciers coutumiers et droits de propriété en Afrique Occidentale, Paris, ORSTOM, Ed. Berger- Levrault, Nouvelle Série n 8, 248 p.

LÉONARD E. et VIMARD P. (2005), Crises et recompositions d'une agriculture pionnière en Côte-d'Ivoire. Dynamiques démographiques et changements économiques dans le Bas-Sassandra, Paris, IRD- Khartala, $361 \mathrm{p}$. 
NYASSOGBO K. G. et al. (1995), Crise économique et mutations socio-démographiques dans une économie de plantation : le cas du Litimé. Programme de Petites Subventions pour la Recherche en Population et développement, Rapport d'étude, 14, UEPA, Dakar, 218 p.

NYASSOGBO K. G. (2003), “Relations ville-campagne et développement local. L'exemple de la petite ville de Badou en zone de plantation au Togo", Les Cahiers d'Outre-Mer. Mis en ligne le 13 février 2008.

OGOUNDÉ L. (1993), “Mutations socio-économiques dans une économie spéculative en crise : le cas des plantations du Litimé au Togo", Annales de l'Université du Bénin, Série Lettres et Sciences Humaines, XIII, pp. 145-189

SAMANI-ZOZO A. (1989), Les aspects juridiques du développement rural en droit togolais, Thèse de doctorat en droit, Faculté de droit et de sciences sociales, Université de Poitiers, 260 p.

SOSSOU K. A. (2000), "L'Akposso et la terre, des origines à nos jours", Actes du colloque du 22 au 25 mars, Lomé, Collection “Patrimoines”, Presses de l’Université du Bénin, pp. 177-192.

TCHAMIÈ T.T.K. (1998), “Facteur humain et évolution des forêts mésophiles de la Région des Plateaux (sud-ouest du Togo)", Actes des Premières Journées Géographiques du Togo, Lomé, du 10 au 13 février 1998, Travaux et Recherches Géographiques, nº spécial, pp. 31-49, Lomé, Faculté des Lettres et Sciences Humaines, Université de Lomé

VERDIER R. et ROCHEGUDE A. (1985), Systèmes fonciers à la ville et à la campagne, Afrique francophone , Paris, Harmattan, 298 p.

\section{NOTES}

1. Le 5 juillet 1884, Gustav Nachtingal signe un traité de protectorat avec le roi Mlapa III de Togoville. A partir de ce moment, le Togo devient une colonie allemande. A la suite de la Première Guerre Mondiale (1914-1918), l'Allemagne est vaincue et perd le Togo (de même que ses autres colonies). C'est ainsi que sa partie occidentale fut confiée à la Grande-Bretagne sous mandat de la S.D.N (actuellement rattachée au Ghana) et la partie orientale à la France (Togo actuel).

2. Il s'agit surtout de la boisson locale sodabi, (distillée à partir du vin de palme) et parfois des liqueurs d'origine étrangère (Schnaps, Royal Stock Gin, Campari, Dubonnet etc.).

3. Cette contrepartie s'élève à 100000 FCFA (Léonard et Vimard, 2005).

4. Ces troubles socio-politiques sont essentiellement dus au difficile début de démocratisation du pays.

5. Selon une étude faite par Adjossou (2009) dans la zone d'étude, l'espace forestier mis en valeur représente $67 \%$ de la superficie totale. Ce taux nous paraît sous-estimé quand on y observe le paysage rural et surtout quand on analyse la carte de végétation. Il faut donc conclure qu'en l'absence de véritables statistiques sur les ressources forestières au Togo, il est difficile de donner un chiffre qui traduit la réalité.

6. Cette expression est de Gu-Konu Y. (1978). 


\section{RÉSUMÉS}

Le dibi-madibi est un mode de faire-valoir indirect qui permit aux populations de s'approprier rapidement l'espace dans le sud-ouest du Togo au début de l'introduction de la culture des plantations de caféiers et de cacaoyers. Ce mode de faire valoir profita aux exploitants venus pour la plupart du nord du pays. Ceux-ci acquirent le droit de propriété sur la moitié des parcelles mises en valeur. Ce droit transmissible ne peut être comparé au plan juridique aux contrats traditionnels connus au Togo. Il ne se confond ni avec le fermage ni avec le métayage classiques. Cette pratique foncière agraire originale crée aujourd'hui un certain nombre de problèmes entre les anciens propriétaires terriens et les exploitants. Les premiers acceptent mal l'acquisition en pleine propriété de la moitié du terrain mis en valeur par les seconds et sont tentés de remettre en cause cette acquisition, ce qui provoque des conflits entre eux.

The dibi-madibi is a mode of tenure that allowed quick indirect ownership of farm space by the population in the south-west of Togo at the beginning of the development of coffee and cocoa plantations. This mode of tenure profited farmers who were mostly from the north of the country. They acquired the ownership right to half of the plot of land that was actually developed. That transmissible right cannot be compared, at the legal level, to the traditional contracts known in Togo. That right should not be confused with either the classical rent or sharecropping. Today, this original agrarian land ownership practice creates a number of problems between the former landowners and the exploiters. The former resent the acquisitionin full ownership of half of the developed land by the latter and are tempted to challenge that acquisition, which causes conflict between them.

\section{INDEX}

Mots-clés : ouest du Togo, faire valoir indirect, droit de propriété, exploitants, propriétaires fonciers, économie de plantation, pleine propriété, dibi-madibi

Keywords : southwestern Togo, indirect farmland ownership, ownership right, exploiters landowners, plantation economy, full ownership

\section{AUTEUR}

\section{PADABÔ KADOUZA}

Département de Géographie, Université de Kara, Togo, kadouza@yahoo.fr 